\title{
Physiological determinants of male mating performance in aphids (Hemiptera: Aphididae)
}

\author{
DiNAH F. HALES \\ Department of Biological Sciences, Macquarie University, NSW 2109, Australia; e-mail: dhales@rna.bio.mq.edu.au
}

\begin{abstract}
Key words. Aphids, Myzus persicae, sexual strategies, sperm economy, ejaculate size, male maturation, sperm availability
\end{abstract}
\begin{abstract}
When investigating sexual strategies of aphids, it is necessary to set up multiple replicates to compensate for the small number of eggs laid per female. Genetic variation among replicates can be minimised if members of the same clone are used, but problems can arise unless the participants are also physiologically equivalent. A series of experiments on Myzus persicae (Sulzer) was performed to investigate aspects of male maturation and semen transfer that should be considered in planning or interpreting experiments on aphid sexual strategies.
\end{abstract}

\section{INTRODUCTION}

The recent explosion of interest in sexual strategies of animals (for example, see reviews by Ball \& Parker, 2000; Jennions \& Petrie, 2000; Bonduriansky, 2001; Zeh \& Zeh, 2001; Pizzari \& Birkhead, 2002; Martin et al., 2004; Snook \& Hosken, 2004) has not surprisingly led aphid workers to begin investigating the strategies used by aphids, in particular sperm economy (Dagg \& Scheurer, 1998; Dagg, 2002, 2003, 2004; Doherty \& Hales, 2002; Doherty et al., 2004). While model insects used to establish basic concepts have high fecundity, their strategies cannot be generalised to cases where females invest their efforts in a small number of large eggs or offspring. For such cases, aphids may have some experimental benefits. An evident advantage is the genetic homogeneity (saving mutation) of all female aphids derived from a single clonal foundress (Blackman, 1987; Sunnucks et al., 1996). Their brothers are also relatively homogeneous, since the males differ only in their possession (at random) of either one of the two X chromosomes carried by their mothers and sisters (Wilson et al., 1997). In the aphid Myzus persicae (Sulzer), for example, the X chromosome is approximately $25 \%$ by length of the haploid genome (Hales et al., 2002). The remaining $75 \%$ of the genome is unequivocally identical between clone brothers (again, excepting mutation) and presumably a large proportion of the two possible Xs is also identical. It should be noted that there is recent evidence of rapid evolution in hypervariable markers in aphids as there is in other organisms (Lushai \& Loxdale, 2002; Loxdale \& Lushai, 2003; Vorwerk et al., 2004) but the majority of changes ( $95 \%$ or more) can be expected to be functionally neutral. The argument for aphids as a model in research on sexual strategies is not weakened by their existence.

However, other aspects of aphid biology make them less favorable as model organisms. The production of mating females and of males is a time-consuming process, requiring four generations in host-alternating aphids such as M. persicae (Hales et al., 1989). The paternity of the eggs needs to be determined by molecular means, though methods (using polymorphic microsatellite markers) have been developed by Wilson for accomplishing this, provided suitable laboratory facilities and expertise are at hand (Sloane et al., 2001; Wilson et al., 2004). Because of the small number of eggs produced by each sexual female ( 10 has been a maximal harvest for $M$. persicae in conditions of our experiments), large numbers of replicates must be set up to obtain sufficient numbers of offspring for analysis. All these features mean that experimental design needs to maximise successful matings. For matings to have a uniform likelihood of success, it is essential to control non-genetic variation among the participants as far as possible. Our experience in attempting to investigate sperm precedence and sperm choice in M. persicae (Doherty \& Hales, 2002; Doherty et al., 2004, Vorburger \& Hales, unpubl.) has suggested some components of age-related variation among males that need to be considered in planning or interpreting experiments on aphid sexual strategies. These components are investigated here. The paper also discusses the anatomy of sperm transfer, since this is relevant to developing a robust model of aphid sperm economy.

\section{MATERIAL AND METHODS}

\section{Aphids, general methods}

The aphids were from genetically-characterised holocyclic clones of Myzus persicae (Sulzer) maintained in the laboratory as synchronised uncrowded cultures on cabbage seedlings (var. Early Jersey Wakefield). Clones used were numbers 31 and 47 (providing males) and 46 and 66 (providing females). Clone 31 had been shown to be more successful than clone 47 at fathering eggs in sperm precedence experiments, though the reason for its success remains unclear (Doherty et al., 2004; Sloane et al., unpubl.). No difference among female clones in readiness to mate has been observed (Vorburger \& Hales, unpubl.).

Stock parthenogenetically-reproducing cultures were kept under banks of fluorescent lights at $20^{\circ} \mathrm{C}$ and a photoperiodic cycle of 16L : 8D. Each host plant was covered with a Perspex cage approximately $4 \mathrm{~cm}$ in diameter and $10 \mathrm{~cm}$ high, the open top being covered with fine gauze material. Sexual individuals were induced as required by transfer of young adult wingless 
parthenogenetic females to a constant temperature room held at $15^{\circ} \mathrm{C}$ and 10L : 14D (lights-on at $09 \mathrm{~h} 00^{\prime}$ ). The offspring of these females, born in the first few days after transfer, were allowed to mature and then distributed 3 per plant to new seedlings. They were then transferred to fresh plants approximately every five days, and produced in succession larvae that would develop as gynoparae (winged females which give birth to sexual females) and winged males (Hales et al., 1989). The gynoparae were placed in groups of three on new seedlings where they gave birth to sexual egg-laying females. This system separated the production of males and females ensuring that they were virgins prior to the experiments. For observation of mating, males and females were removed from plants to Petri dishes $(3.5 \mathrm{~cm}$ in diameter) containing a square of moistened filter paper, with one female and one male per arena. These experiments were carried out at $20^{\circ} \mathrm{C}$, between about $11 \mathrm{~h} 00^{\circ}$ and $18 \mathrm{~h} 00^{\prime}$. All aphids used were less than 10 days into adulthood at the start of mating trials (Doherty \& Hales, 2002) and no intraclonal matings were permitted.

Aphids were dissected in Blackman's aphid saline (Blackman et al., 1995) using a Zeiss stereomicroscope. Dissected spermathecae were transferred to microscope slides in a drop of saline, examined using phase contrast with an Olympus Vanox microscope, and measured with an eyepiece micrometer. Size of the spermatheca, presence, activity and appearance of contained sperm were noted. The volume of the spermatheca was calculated by the formula $4 / 3 \pi r_{1}^{2} r_{2}$ where $r_{1}$ is the shorter and $r_{2}$ the longer radius, i.e. simplifying the shape to an ellipsoid. Aphids were weighed on a Cahn 25 Electrobalance. Statview for Macintosh and Microsoft Excel were used for calculating statistics.

\section{Male maturation}

During earlier experiments, it was observed that males in early adulthood were unlikely to mate (Hales, unpubl. observ.; cf. Doherty \& Hales, 2002). In M. persicae in our experimental conditions, a good sign of mating readiness was the migration of recently-ecdysed adult males from the plant to the roof of the cage. The delay after adult ecdysis until migration and willingness to mate were determined for a sample of males (14 from clone 31,18 from clone 47 ).

It had also been observed that very heavy males (mass $>300$ $\mu \mathrm{g})$ were unlikely to mate (Hales, unpublished observations). Further, heavier males were less successful than lighter ones in fathering eggs when two males mated with a female, though this effect was confounded with clone, since males of clone 47 were usually heavier than those of clone 31 (Doherty et al., 2004) Because of the possible correlation between body mass and maturation towards mating readiness, body mass was measured from ecdysis to migration, and for a sub-sample, in more detail over the first six hours from ecdysis.

\section{Anatomy of sperm transmission}

Reproductive systems were dissected from male aphids and mounted for phase contrast microscopy to determine the appearance of sperm and its stage of development for comparison with other published accounts.

Mated and virgin spermathecae were dissected out and photographed, using bright field or differential interference contrast microscopy. Placement of the aedeagus was inferred from the distribution of sperm in the female reproductive system shortly after mating. Observations of spermatheca size in females mated repeatedly were also made, by placing five virgin females of clone 46 in separate arenas and introducing three virgin males of clone 31 in succession to each, the new male replacing the previous one as soon as the first mating was completed.
Dagg (2002) interpreted the failure of old virgin females to mate (see also Doherty \& Hales, 2002) to be due to the presence of dried honeydew around the genital region and suggested that this would apply also in M. persicae. Observations were therefore made of old $M$. persicae females (about three weeks after adult ecdysis).

\section{Sperm availability}

To determine whether males can successfully transfer sperm and fertilise eggs in repeated matings, two experiments were devised.

Experiment 1. Fifteen individual males of clone 31 were placed with single virgin females of clone 66 in Petri dish arenas. Once copulation had ended, the mated female was replaced with another virgin, and so on for up to four successive copulations. As many mated females as possible were dissected for observation and measurement of the spermatheca, while the other females were returned to plants to reproduce. Dissection was done at least one hour after mating. The males were also returned singly to plants and were allowed successive copulations again $48 \mathrm{~h}$ after the first series and yet again after a further $48 \mathrm{~h}$. The experiment quickly generated more mated females than could be dissected, so a simpler one was designed as below.

Experiment 2. Five individual males of clone 31 were mated singly with virgin females of clone 66 as described above, but were permitted only three successive copulations per day for a period of five successive days. Delay before copulation and duration of copulation were recorded. Surviving males were again introduced to females on the $8^{\text {th }}$ day, but could not be given further opportunities because insufficient females were available. All females from the first three days were dissected and those from the later matings were placed individually on plants for a functional test of their ability to lay fertilised eggs, which are readily identified by their glossy black colour within a few days after laying. Eggs were checked 2 weeks after mating.

\section{RESULTS}

\section{Male maturation}

Following ecdysis, adult males remained on the plant for between 20 and $28 \mathrm{~h}$. There was then a strong tendency for them to leave the plant during the later part of the photoperiod, i.e. between 2 and 4 pm, or 5-7 h after lights on (22 out of 23 observations; 6 other individuals migrated outside the observation period). Males given access to females before or immediately after migration did not mate. Some aphids given access to females $20 \mathrm{~h}$ after migration did not mate, but $4 \mathrm{~h}$ later they mated rapidly. Hence a delay of as much as $48 \mathrm{~h}$ from ecdysis to mating should be allowed to ensure mating readiness, in the conditions of these experiments.

During the period before migration, males lost 35-40\% of their body mass (paired t-test, $\mathrm{p}<0.0001$ ). The loss was linear during the first $6 \mathrm{~h}$ but slowed overnight. Previous experiments (Sloane \& Hales, unpubl.) had indicated that newly-ecdysed sexual females did not lose significant body mass.

\section{Anatomy of sperm transmission}

Sperm in the follicles and vasa deferentia were not joined at the heads (cf. Dagg, 2002) and were generally not motile. Motility was sometimes noted in sperm in the 


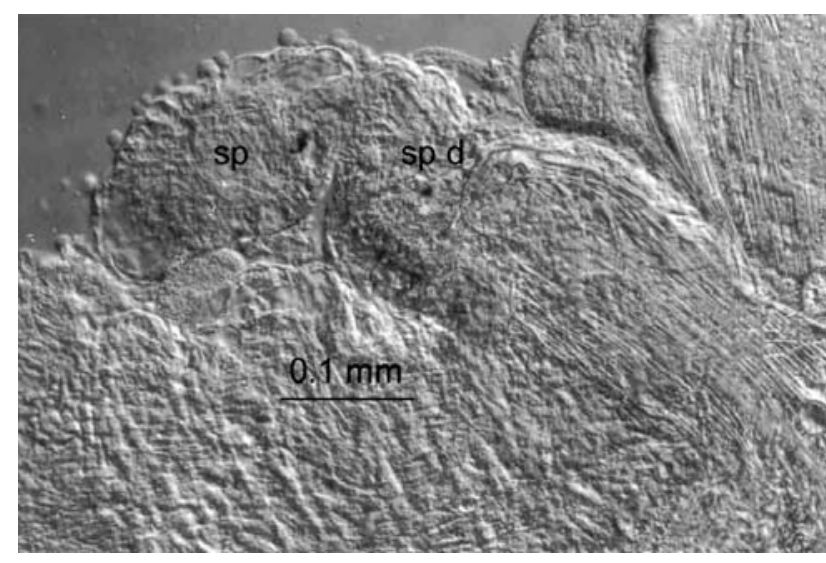

Fig. 1. Spermatheca of unmated adult female of Myzus persicae, mounted in saline and photographed with differential interference contrast microscopy. $\mathrm{Sp}=$ spermatheca, $\mathrm{sp} \mathrm{d}=$ spermathecal duct.

vasa deferentia, in the part distal to the testis, and in sperm released during dissection, perhaps following contact with accessory gland secretion. Length of released sperm was approximately $0.32 \mathrm{~mm}$.

The appearance of the spermatheca in virgin and mated females is shown in Figs 1 and 2. The round objects in the spermatheca (Fig. 2) are likely to be an optical artefact related to the swirling of sperm near the spermathecal duct (see below). In contrast Dagg (2002) showed round bundles with the heads facing inwards. Previously published illustrations of the reproductive system in sexual female aphids fail to show the long, narrow, angled approach to the spermatheca by way of the spermathecal duct. This can be seen in Fig. 2.

During copulation, the tip of the aedeagus is most likely in the spermathecal duct. Its termination in the duct, rather than the spermatheca, was inferred from females dissected within an hour of mating: their spermathecal ducts were all distended with sperm. The shortest period from commencement of mating to observation of the slide-mounted spermatheca was $20 \mathrm{~min}$; in these females some sperm had already reached the spermatheca. Sperm within the duct were motile, but the most dramatic movement towards the spermatheca was by mass flow, seemingly induced by influx of fluid into the duct. Sperm entering the spermatheca in these conditions formed a 'whirlpool' near the opening of the spermathecal duct. Where mass flow was not observed, contractions of the duct rather than their own movement seemed to provide the main force for transfer of sperm to the spermatheca. Sperm could be seen gradually moving around the periphery of the spermatheca. The mass flow was perhaps an artefact of dissection, but this cannot be confirmed since in vivo observations in intact specimens are impossible.

Spermatheca size in females mated successively with three virgin males averaged $0.0074 \mathrm{~mm}^{3}(\mathrm{n}=3)$. This was larger than the mean for any group of females mated once by virgin males $(0.0044, \mathrm{n}=7 ; 0.0052, \mathrm{n}=9 ; 0.0052, \mathrm{n}=$

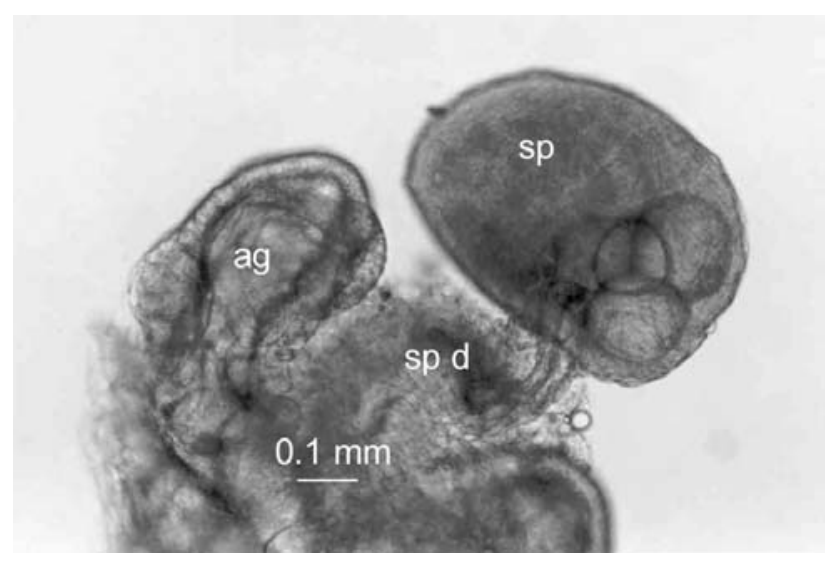

Fig. 2. Spermatheca of mated adult female of Myzus persicae, mounted in saline and photographed with bright field microscopy. $\mathrm{Sp}=$ spermatheca, $\mathrm{sp} \mathrm{d}=$ spermathecal duct, a $\mathrm{g}=$ accessory gland.

9; $0.0025, \mathrm{n}=5$ ). The maximum calculated volume from a single mating was $0.0096 \mathrm{~mm}^{3}$.

Old virgin females became swollen with unlaid eggs. The genital plate was frequently dirty with adhering materials (soil grains or fungi). There was no evidence of dried honeydew.

\section{Sperm availability}

The first copulation by most males in experiment 1 was longer than the subsequent ones and ranged from 15 to 61 min (mean $28.25 \mathrm{~min}, \mathrm{n}=6$ ). The mean duration for later copulations was 14-16 min (range 2-35). No correlation was seen between delay before copulation and duration of copulation, or copulation duration and spermathecal volume (except where matings were very short, with little or no semen transfer). Mean duration of copulation showed virtually no change from a male's first to his $18^{\text {th }}$ mating in experiment 2. Almost all copulations lasted between 12 and $22 \mathrm{~min}$. The delay before copulation, from introduction of a male to the female's arena, showed no consistent pattern with repeated matings in either experiment.

Males transmitted less semen on average, as judged by the volume of the spermatheca, from the first to later matings with successive females, but a regular decrease was not observed for males taken individually. The mean spermathecal size showed a decline with mating number, with a slope of -18 in the first experiment and -19 in the

TABLE 1. Increase in ejaculate volume from males rested between mating series (Experiment 2).

\begin{tabular}{cccc}
\hline \multirow{2}{*}{ Male \# } & \multicolumn{3}{c}{ Spermathecal volume in $\mathrm{mm}^{3} \times 10^{5}$, distributed } \\
\cline { 2 - 4 } & Day 1 & Day 2 & Day 3 \\
\hline 1a & $169,208,214$ & $296,133,85$ & Not measured \\
2 & $131,290,132$ & 290 & 97 \\
3 & $625,124,124$ & 136,82 & $80,91,82$ \\
4 & (missing), 268, 91 & $121,174,163$ & $130,39,71$ \\
5 & $67,139,69$ & $165,55,67$ & 54,71, (no sperm) \\
\hline
\end{tabular}


TABLE 2. Increase in ejaculate volume from males rested between mating series (Experiment 1) (? Indicates data not included because first female may have mated twice).

\begin{tabular}{cccc}
\hline \multirow{2}{*}{ Male \# } & \multicolumn{3}{c}{$\begin{array}{c}\text { Spermathecal volume in } \mathrm{mm}^{3} \times 10^{5} \text {, distributed } \\
\text { by day of mating }\end{array}$} \\
\cline { 2 - 4 } & Day 1 & Day 3 & Day 5 \\
\hline 3 & $?, 121$ & 469 & 21 \\
4 & $?$ & 192,115 & 97,151 \\
8 & Not measured (2) & 104,77 & 174,77 \\
9 & $181,232,156$ & 113,100 & 278 \\
11 & Not measured (2) & 121,31 & 211 \\
12 & 304,208 & 212,155 & \\
15 & $214,26,844,242$ & 296 & \\
\hline
\end{tabular}

second, where spermathecal volume was expressed as $\mathrm{mm}^{3} \times 10^{5}$. In Experiment 2, there was some evidence of an overnight recovery in semen volumes after the first day's matings, but this did not occur after the second day (Table 1). Each of the males had mated three times on the first day, then had been isolated overnight. The increase in ejaculate volume from the last mating on day 1 to the first on day 2 approached significance (paired sign test, $p$ $=0.0625$ ). The incomplete data for Experiment 1 (Table 2) support this evidence for recovery (in six out of nine cases ejaculate volume was greater after each 2 day recovery period). The recovery indicates that a regression line for semen volume on mating number gives only a coarse-grained picture of sperm availability. Two males in Experiment 2 transferred sufficient sperm to fertilise eggs at mating number 18 , but for each of them three earlier matings had been unsuccessful. Four out of five aphids successfully transferred sperm in all their first 8 matings, the other aphid being unsuccessful at mating 7. Any mating in which sperm were transferred provided a large excess of sperm beyond that required to fertilise all the eggs that a female might potentially lay (up to 40 , estimated from a count of egg load in old virgin females, Dagg, 2002).

\section{DISCUSSION}

The experiments reported here have demonstrated some important aspects of male aphid biology that need consideration in designing experiments to elucidate sexual strategies of aphids.

- Male aphids require a maturation period after ecdysis to the adult stage before they can mate - up to $48 \mathrm{~h}$ in M. persicae in the conditions of these experiments.

- The time of migration from the host plant occurs preferentially at a certain period of the light-dark cycle.

- Male aphids lose substantial body mass between ecdysis and mating readiness.

- Sperm maturation is not uniform across aphid species.

- Male aphids can transmit sperm and father eggs for many copulations when mated with successive females.

- There is a decline in the average volume of ejaculate from first to later matings, but a recovery in ejaculate volume may occur after a rest period.
- Failure of old females to mate is not necessarily related to presence of dried honeydew.

The period of time from adult ecdysis to migration and mating readiness is an important consideration in selecting males of $M$. persicae (or other species where males disperse) for mating experiments. In planning large experiments on sexual strategies in aphids, species which do not host-alternate would have considerable advantages, and these may have different dynamics. Paternity success in some insects is related to body size; understanding the relationship between time from ecdysis, body mass and mating readiness in aphids will avoid false correlation between body mass and paternity success. A linear measurement such as hind tibia length may be a more reliable parameter for determining any effect of male size on female post-copulatory sperm choice, though females might be intuitively expected to identify the body mass of their mating partners more readily as a fitness indicator.

Dagg $(2002,2003)$ has recently published some observations on male aphid behaviour and physiology as background to his theoretical thesis on sexual strategies. In particular he compared his interpretations of sperm economy in the large thistle aphid [Uroleucon cirsii (L.)] with one of the classic "models" of optimal mating theory, the yellow dung fly, Scathophaga stercoraria (L.), (e.g. Simmons et al., 1999). Some a priori assumptions underlying discussions of sperm economy include unlimited sperm resource in males, instant mixing in the female's store, sperm displacement and "last male precedence" (provided copula duration is equal in successive matings). Dagg $(2002,2003)$ showed that copula duration in successive matings was not equal in the thistle aphid and hypothesised that limitations on sperm availability and on female capacity to store sperm were variations from the dung-fly model. He suggested that sperm precedence might occur through the second male's sperm being positioned close to the outlet from the spermatheca, i.e. lack of mixing.

Sperm precedence is said to occur if the last male to mate with a female fathers most of her eggs. It is often mechanistically based on the last male's ability to displace sperm from the spermatheca, or to redistribute it so that his own sperm are more likely to fertilise eggs, as occurs in Odonata (Cordoba-Aguilar et al., 2003). We have tested the possibility that aphids exhibit sperm precedence (Doherty \& Hales, 2002, Doherty et al., 2004). However, a strong effect of male genotype confounded our experiments, males of clone 31 always fathering more eggs than males of two other clones (47 and 66), regardless of mating order. Clone 47 males are fertile, and as willing to mate as clone 31 (Doherty et al. 2004, Sloane \& Hales, in prep.), so the explanation of clone 31 's dominance must be sought within the female reproductive tract. Sperm competition or female sperm choice may thus be important components in aphid sexual strategies.

Semen is deposited initially in the spermathecal duct, and hence males are unlikely to displace semen from a 
previous mating if it has already reached the spermatheca. However, a second mating might physically displace sperm still in the spermathecal duct. The limits to elasticity of the spermatheca most likely prevent the deposition of additional semen after a female has stored sperm from a small number of matings. The spermatheca becomes turgid and translucent, suggesting that its wall is fully stretched. This appearance may be attained after a single mating (the maximum volume calculated after a single mating was $0.0096 \mathrm{~mm}^{3}$ ) but most single matings transferred less semen, so that 3-4 males might successfully inseminate a female. The swirling masses of newlyentering sperm near the entry from the spermathecal duct tend to support Dagg's contention (Dagg, 2002, 2003) that new sperm accumulate in this area and mixing is slow. However, the observation that clone 31 males father more eggs than other clones, regardless of mating order, suggests that a spermathecal process other than "last-infirst-out" determines which sperm successfully fertilise eggs. At present there is no evidence that sperm precedence occurs in aphids, and attention may be better directed towards investigating sperm competition or cryptic sperm choice by females. The present evidence indicates, in accordance with previous hypotheses of Dagg $(2002,2003)$ that storage within the female and production in the male aphid are to some extent limiting. Nevertheless far more sperm can be stored (and are transmitted at any mating) than needed for fertilisation of all the eggs a female might lay. Equally, a male has the ability to inseminate a relatively large number of females. However the decline in semen volume from first to later matings would favour paternity by previously virgin males, on simple stochastic grounds, in cases where a virgin male and one which has mated several times both fertilise a given female.

Dagg (2002) showed that sperm deposited within the spermathecal duct of females in the large thistle aphid $U$. cirsii were still joined at the head and speculated that they might reach the spermatheca as 'cooperating bundles'. In M. persicae the sperm observed even within the testis of adult males were not joined at the head, though they were during the last nymphal stage. They were not motile within the testis, but became motile when released from the vas deferens during dissection, possibly on contact with accessory gland secretion. In contrast, motile sperm within the testes of Sitobion spp. have been observed (Hales, unpubl. observ.). Work on other insects suggests that a period of capacitance within the spermatheca may be necessary before sperm are able to fertilise eggs (Longo et al., 1993). It is impossible to generalise across species about aphid sperm maturation from the small number of observations recorded to date.

A point of contrast with Dagg's observations concerns the failure of old females to mate: in $M$. persicae there was no sign of dried honeydew occluding the genital opening. Soil grains or fungi adhering to accessory gland secretion on the genital plate may give false signals to males, or the swollen, egg-filled abdomens of older unmated females may make mounting difficult. It is clear that more work should be done on signals, other than pheromonal, that initiate mating in aphids.

While not systematically investigated, post-copulatory guarding of females by $M$. persicae males has frequently been observed during these experiments. Dagg (2004) pointed out the theoretical expectation that aphids with guarding would have less female-biased sex ratios than those without: this contention is not supported by M. persicae laboratory data (Hales et al., 1989, 1996). Since the sex ratio of aphids in laboratory experiments is overwhelmingly influenced by the photoperiodic regime, field observations are in some respects more realistic. Daiber $\&$ Schöll (1959) found very few males of $M$. persicae on the primary host with weekly sampling at several sites in South Africa.

One aspect of male physiology neglected in these experiments is the possible existence of circadian rhythms of male responsiveness to female sex pheromones. Females have a known circadian cycle of pheromone release from glands on the hind tibiae (see for example Guldemond et al., 1994; Guldemond \& Dixon, 1994; Thieme \& Dixon, 1996, Goldensaz \& McNeil, 2003). The experiments described in the present paper were probably unaffected by pheromone attraction, since the aphids were confined in small containers which would become saturated with pheromone if it was released. General observations suggested no orientation towards the female: mating took place after random encounter and was more likely to occur when males were actively walking around the container. Consideration of potential effects of rhythms of pheromone release and responsiveness may be important in experiments using different configurations, and in particular, in field experiments or attempts at interspecific hybridisation.

Studies of aphid sexual strategies are in their infancy, and all the parameters noted above need further work in a range of species. Aspects of female choice, ranging from mate acceptance to sperm utilisation or possible sperm dumping, remain virtually untouched. It is suggested that monoecious aphids (i.e. aphids which do not have obligatory host alternation) will provide logistically simpler experimental systems initially and will result in larger numbers of eggs per mating.

ACKNOWLEDGEMENTS. I thank the Department of Biological Sciences, Macquarie University, for access to facilities. I thank R. Oldfield for photographing the spermathecae, and P. Sunnucks (La Trobe University) for advice on statistical methods.

\section{REFERENCES}

Ball M.A. \& Parker G.A. 2000: Sperm competition games: a comparison of loaded raffle models and their biological implications. J. Theor. Biol. 206: 487-506.

BLACKMAN R.L. 1987: Reproduction, cytogenetics and development. In Minks A.K. \& Harrewijn P. (eds): Aphids, their Biology, Natural Enemies and Control. Vol. 2A. Elsevier, Amsterdam, pp. 163-195.

Blackman R.L., Spence J.M., Field L.M. \& Devonshire A.L. 1995: Chromosomal location of the amplified esterase genes 
conferring resistance to insecticides in the peach-potato aphid Myzus persicae. Heredity 74: 297-302.

BONDURIANSKY R. 2001: The evolution of mate choice in insects: a synthesis of ideas and evidence. Biol. Rev. 76: 305-339.

Cordoba-Aguilar A., Uhia E. \& Rivera A.C. 2003: Sperm competition in Odonata (Insecta): the evolution of female sperm storage and rivals' sperm displacement. J. Zool. 261: 381-398.

DAGg J. 2002: Strategies of Sexual Reproduction in Aphids. PhD thesis, University of Göttingen, Germany. [http://webdoc.sub.gwdg.de/diss/2002/dagg/dagg.pdf].

DAGG J.L. 2003: Copula duration and sperm economy in the large thistle aphid, Uroleucon cirsii (Hemiptera:Aphididae). Eur. J. Entomol. 100: 201-203.

DAGG J.L. 2004: Post-copula guarding and sex ratios in aphids. In Simon J.-C., Dedryver C.-A., Rispe C. \& Hullé M. (eds) Aphids in a New Millennium, Proceedings of 6th International Symposium on Aphids, Rennes, France, 2001. INRA, Versailles, pp. 33-37.

DAGG J. \& SCHEURER S. 1998: Observations on some patterns of males' sexual behaviour of certain aphid species indicate the existence of male marking pheromones. In Nieto Nafría J.M. \& Dixon A.F.G. (eds): Aphids in Natural and Managed Ecosystems. Universidad de León, León, Spain, pp. 167-171.

DAIBER C.C. \& SCHÖLL S.E. 1959: Further notes on the overwintering of the green peach aphid, Myzus persicae, in South Africa. J. Entomol. Soc. S. Africa 22: 494-520.

DoherTy H.M. \& HaLEs D. 2002: Mating success and mating behaviour of the aphid Myzus persicae (Hemiptera: Aphididae). Eur. J. Entomol. 99: 23-27.

Doherty H., Hales D. \& Sloane M. 2004: Male mating performance in Myzus persicae (Sulzer). In Simon J.-C., Dedryver C.-A., Rispe C. \& Hullé M. (eds): Aphids in a New Millennium, Proceedings of 6th International Symposium on Aphids, Rennes, France, 2001. INRA, Versailles, pp. 207-212.

Hales D.F., Wellings P.W. \& Parkes R.A. 1989: Investment in gynoparae and males by Myzus persicae (Sulzer). Funct. Ecol. 3: 727-734.

Hales D.F., Tomiuk J., Wöhrmann K. \& Sunnucks P. 1996 Evolutionary and genetic aspects of aphid biology: a review. Eur. J. Entomol. 94: 1-55.

Hales D.F., Wilson A.C.C., Sloane M.A., Simon J.-C., Le Gallic J.-F. \& SunNucKs P. 2002: Lack of detectable genetic recombination on the $\mathrm{X}$ chromosome during the parthenogenetic production of female and male aphids. Genet. Res., Camb. 79: 203-209.

Goldensaz S.H. \& McNeil J.N. 2003: Calling behaviour of the potato aphid Macrosiphum euphorbiae oviparae under laboratory and field conditions. Ecol. Entomol. 28: 291-298.

GuldEMOND J.A. \& Dixon A.F.G. 1994: Specificity and daily cycle of release of sex pheromones in aphids: a case of reinforcement? Biol. J. Linn. Soc. 32: 287-303.

Guldemond J.A., Tigges W.T. \& DE VRIJer P.W.F. 1994: Circadian rhythm of sex pheromone production and male activity of coexisting sibling species of Cryptomyzus aphids (Homoptera: Aphididae). Eur. J. Entomol. 91: 85-89.

Jennions M.D. \& Petrie M. 2000: Why do insects mate multiply? A review of the genetic benefits. Biol. Rev. 75: 21-64.

Longo G., Sottile L., Viscuso R., Giuffrida A. \& Profitera R. 1993: Ultrastructural changes in sperm in Eyprerepocnemis plorans (Charpentier) (Orthoptera: Acrididae) during storage of gametes in the female genital tract. Invert. Reprod. Dev. 24: $1-6$.

LoXDALE H.D. \& Lushai G. 2003: Rapid changes in clonal lines: the death of a sacred cow. Biol. J. Linn. Soc. 79: 3-16.

LuSHAI G. \& LOXDALE H.D. 2002: The genetic improbability of a clone. Genet. Res., Camb. 79: 1-9.

Martin O.Y., Hosken D.J. \& Ward P.I. 2004: Post-copulatory sexual selection and female fitness in Scathophaga stercoraria. Proc. R. Soc. Lond. (B) 271: 353-359.

PizZARI T. \& BiRKHEAD T.R. 2002: The sexually-selected sperm hypothesis: sex-biased inheritance and sexual antagonism. Biol. Rev. 77: 183-209.

Simmons L.W., Parker G.A. \& Stockley P. 1999: Sperm displacement in the yellow dung fly, Scatophaga stercoraria: and investigation of male and female processes. Am. Nat. 153: 302-314.

Sloane M.A., Sunnucks P., Wilson A.C.C. \& Hales D.F. 2001: Microsatellite isolation, linkage group identification and determination of recombination frequency in the peach-potato aphid, Myzus persicae (Sulzer) (Hemiptera: Aphididae). Genet. Res., Camb. 77: 251-260.

SNOOK R.R. \& Hosken D.J. 2004: Sperm death and dumping in Drosophila. Nature 428: 939-941.

Sunnucks P., England P.R., Taylor A.C. \& Hales D.F. 1996: Microsatellite and chromosome evolution of parthenogenetic Sitobion aphids in Australia. Genetics 144: 747-756.

Thieme T. \& Dixon A.F.G. 1996: Mate recognition in the Aphis fabae complex: daily rhythm of release and specificity of sex pheromones. Entomol. Exp. Appl. 79: 85-89.

Vorwerk S., Blaich R., Brunner H. \& Forneck A. 2004: Genetic variation within parthenogenetic lineages - adaptational and mutational processes. Abstract, PARTNER-3 Workshop, University of Münster, October 2004.

Wilson A.C.C., Massonet B., Simon J.-C., Prunier-Leterme N., Dolatti L., Llewellyn K.S., Figueroa C., Ramirez C., Blackman R.L., Estoup A. \& Sunnucks P. 2004: Crossspecies amplification of microsatellite loci in aphids: assessment and application. Mol. Ecol. Notes 4: 104-109.

Wilson A.C.C., Sunnucks P. \& Hales D.F. 1997: Random loss of $\mathrm{X}$ chromosome at male determination in an aphid, Sitobion $\mathrm{nr}$ fragariae, detected using an X-linked polymorphic microsatellite marker. Genet. Res., Camb. 69: 233-236.

Zен J.A. \& Zен D.W. 2001: Reproductive mode and the genetic benefits of polyandry. Anim. Behaviour. 61: 1051-1063.

Received February 11, 2005; revised and accepted April 15, 2005 